\title{
Comportamento de compra dos consumidores de frutas, legumes e verduras na região central do Rio Grande do Sul
}

\author{
Purchase behaviour of fruit and vegetable consumers in the central region of \\ Rio Grande do Sul
}

\author{
Renato Santos de Souza ${ }^{I^{*}}$ Alessandro Porporatti Arbage ${ }^{\mathrm{I}}$ Pedro Selvino Neumann ${ }^{\mathrm{I}}$ \\ José Marcos Froehlich ${ }^{\mathrm{I}}$ Vivien Diesel ${ }^{\mathrm{I}}$ Paulo Roberto Silveira' ${ }^{\mathrm{I}}$ Alexandre da Silva ${ }^{\mathrm{II}}$ \\ Cristiano Corazza ${ }^{\text {III }}$ Edner Baumhardt ${ }^{\text {IV }}$ Rodrigo da Silva Lisboa ${ }^{\text {II }}$
}

\section{RESUMO}

O objetivo deste artigo é identificar as preferências dos consumidores de frutas, legumes e verduras (FLV) em termos de estabelecimentos de varejo por eles escolhidos, hábitos de consumo e principais critérios considerados em suas decisões de compra. Do ponto de vista metodológico, a pesquisa conduzida trata-se de um survey (levantamento), realizado em 11 municípios da região central do Rio Grande do Sul. Foram entrevistados 266 consumidores acima de 18 anos, que compuseram uma amostra sistemática e estratificada por região, sexo, idade e renda. Concluiu-se que a maioria dos consumidores adquire os produtos em supermercados ou hipermercados, sendo que a freqüencia de compra de maior incidência identificada foi entre duas e três vezes por semana. Para a escolha do estabelecimento a ser utilizado, os consumidores levam em conta, preferencialmente, qualidade, variedade de produto e a agilidade no check-out. Este resultado indica a importância primordial de dois aspectos quando da escolha de FLV, do ponto de vista dos consumidores: qualidade de produto e tempo dedicado para a compra. Da mesma forma, os dados indicam que, dos cinco principais aspectos considerados pelos consumidores para a escolha de FLV, quatro referem-se à qualidade (aparência, sabor, aspectos nutricionais e durabilidade), aparecendo o preço apenas em terceiro lugar. Por fim, a pesquisa identificou três grupos de produtos segundo a regularidade de consumo, dos quais o conjunto de produtos consumidos de forma mais regular é composto por: cebola, laranja, tempero verde, tomate, alho e alface.

Palavras-chave: pesquisa de mercado, comportamento de compra, hortigranjeiros.

\begin{abstract}
This article is aimed at identifying the preferences of fruit and vegetable (FLV) consumers concerning the place of purchase, eating habits and main criteria used during purchase. The study was carried out through a survey made in 11 counties in the central region of Rio Grande do Sul. Two hundred sixty six consumers aged over 18 years-old were interviewed and constituted a systematic sampling stratified according to region, sex, age and income. It was concluded that most of the consumers acquire these products in supermarkets, about two or three times a week, in most cases. When choosing the place of purchasing the consumers consider, mainly, quality, variety and speed at the check-out. This result indicates that there are two main aspects when choosing FLV, from the consumers' point-of-view: quality of the product and time dedicated in making the purchase. In the same way, the data indicates that out of the five main aspects considered by the consumers when choosing FLV, four refer to the quality (appearance, flavour, nutritional aspects and durability), being that the price appears only in third place. Finally, this study identified three groups of products according to purchase frequency, out of which, the group with the highest frequency is composed by: onions, oranges, parsley, tomatoes, garlic and lettuce.
\end{abstract}

Key words: market survey, purchase behaviour, horticulture.

\section{INTRODUÇÃO}

Nas últimas três décadas, a sociedade tem passado por transformações com reflexos diretos e

IDepartamento de Educação Agrícola e Extensão Rural, Centro de Ciências Rurais (CCR), Universidade Federal de Santa Maria (UFSM). Prédio 44, Faixa de Camobi, km 09, 97105-900, Santa Maria, RS, Brasil. E-mail: rssouza@smail.ufsm.br. *Autor para correspondência.

IICurso de Pós-graduação em Extensão Rural, UFSM, Santa Maria, RS, Brasil.

${ }^{\mathrm{III} C o o p e r a t i v a ~ A g r i ́ c o l a ~ d e ~ E s p u m o s o, ~ E s p u m o s o, ~ R S, ~ B r a s i l . ~}$

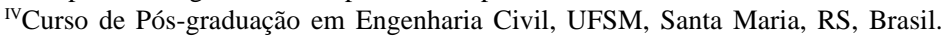


indiretos sobre o estilo de vida e os hábitos de consumo da população. Essas mudanças podem ser exemplificadas pelo aumento da freqüência de alimentação fora da residência, maior participação da mulher no mercado de trabalho, maior urbanização, aumento da busca por qualidade de vida e assim por diante (PAULILLO \& PESSANHA, 2002).

Do ponto de vista da demanda e do padrão de consumo alimentar, as mudanças recentes têm apontado para três tendências básicas: menor passividade, maior conscientização e exigência dos consumidores frente à oferta de produtos alimentícios; crescente importância de atributos de qualidade (saúde, ecologia, etc.) associados a algum tipo de serviço ou facilidade na decisão dos consumidores finais; e avanço das grandes redes varejistas, com ampliação da oferta de produtos, inclusive das suas próprias marcas (JUNIOR, 1999; KOHLS, 2004).

Diversas pesquisas dão conta de que os hábitos de consumo se deslocam no sentido da preferência por qualidade em um sentido amplo, considerando, além dos aspectos intrínsecos ao produto, questões relacionadas à logística, à sanidade e a impactos sociais e ambientais gerados a partir dos processos produtivos (JUNIOR, 1999; KOHLS, 2004).

Dentro desse contexto de reorganização dos processos de produção e comercialização de alimentos, ressalta-se a importância de estudar como ocorre o processo de compra de FLV, considerando ser este um mercado em constante expansão e sem ainda um direcionamento claro sobre os seus principais critérios competitivos.

Dessa forma, o presente estudo pretende contribuir para a compreensão da natureza de funcionamento dos mercados agrícolas ao analisar o comportamento de compra dos consumidores finais de FLV na região central do Estado. O principal objetivo é identificar as preferências dos consumidores em termos de escolha por estabelecimentos de varejo para suas compras, seus principais hábitos de consumo e os critérios utilizados no momento de comprar FLV. A inexistência de pesquisas de mercado desta natureza nesta região do Rio Grande do Sul permite justificar a importância deste tipo de estudo, que pode orientar, de forma mais consistente e segura, as estratégias produtivas deste segmento de produtos.

Os resultados aqui relatados fazem parte de um projeto de pesquisa financiado pela FAPERGS (Fundação de Amparo à Pesquisa do Rio Grande do Sul), que visa a estabelecer um diagnóstico de produção e de consumo de FLV e Produtos Coloniais na região central do Rio Grande do Sul.

\section{MATERIAL E MÉTODOS}

O presente trabalho pode ser descrito como um survey, ou levantamento, que, conforme KERLINGER (1980), é um tipo de pesquisa que busca estudar pequenas e grandes populações utilizando amostras, com o objetivo de descobrir a incidência relativa, distribuição e/ou inter-relação de variáveis. O propósito desse tipo de estudo, de acordo com FOWLER (1993), é produzir estatísticas, isto é, resultados quantitativos de alguns aspectos de uma população estudada, que, no caso aqui apresentado, referem-se ao comportamento de compra de FLV por parte dos consumidores urbanos.

A área de abrangência do estudo compreende 11 municípios localizados na região central do Rio Grande do Sul: Santa Maria e Cachoeira do Sul (principais centros consumidores regionais), juntamente com a região denominada de Quarta Colônia de Imigração Italiana (importante produtora de FLV da região), da qual fazem parte os municípios de Silveira Martins, São João do Polêsine, Faxinal do Soturno, Ivorá, Nova Palma, Agudo, Dona Francisca, Restinga Seca e Pinhal Grande.

Santa Maria e Cachoeira do Sul são os municípios mais populosos dentre os pesquisados, com 243.611 e 87.873 habitantes, respectivamente. Por outro lado, a Quarta Colônia é formada por pequenos municípios e com população total de 63.443 habitantes. No total, portanto, a população alvo da pesquisa compreende um universo de 394.927 habitantes (IBGE, 2000).

As entrevistas foram realizadas apenas com pessoas maiores de 18 anos, tendo sido entrevistados 266 consumidores, dos quais 176 em Santa Maria, 55 em Cachoeira do Sul e 35 na Quarta Colônia. O tamanho da amostra representa aproximadamente 0,1\% da população dessa faixa etária, o que foi considerado adequado e representativo face às características da pesquisa, aos procedimentos amostrais utilizados e as possibilidades operacionais de trabalho.

A amostra realizada pode ser descrita como estratificada e sistemática. Para garantir a maior representatividade da amostra e sua aproximação com o perfil da população, foram respeitados critérios de estratificação por região (no caso de Santa Maria e Cachoeira do Sul, inclusive por região da cidade), sexo, idade e faixas de renda, com base em dados da população disponíveis no Instituto Brasileiro de Geografia e Estatística (IBGE).

O procedimento de estratificação permite subdividir a população em segmentos mais homogêneos, o que possibilita que amostras de 
menores dimensões sejam satisfatoriamente representativas dessa população (ADLER, 1971).

Nos locais de realização das entrevistas, por sua vez, os entrevistados foram escolhidos por um procedimento sistemático (contagem de um número fixo de pessoas que passavam à frente do local estabelecido para as entrevistas, selecionando a seguinte), que garantiu o não-viés da amostra.

As entrevistas ocorreram nas sedes dos municípios, em locais neutros (sem maior proximidade com bancos, paradas de ônibus, supermercados, etc., também para se evitar viés na amostra) e de grande fluxo de pessoas, normalmente no centro das cidades.

O questionário era composto de 28 questões distribuídas em duas partes: uma primeira, que buscava avaliar o comportamento de compra do consumidor, e uma segunda parte que objetivava descrever algumas características do entrevistado, através de dados demográficos, de estilo de vida e aspectos de natureza cultural.

A formulação do questionário seguiu o Modelo de Gains (GAINS, 1994), para quem o comportamento de escolha e compra de alimentos resulta da interação de três fatores: o alimento em si (aparência, cheiro, valor nutricional, sabor, etc.), o consumidor (aspectos culturais, estilo de vida e demografia) e o contexto (onde e de que forma o consumidor adquire os produtos, quando ou com que periodicidade, etc.) (LIMA FILHO et al., 2004). Informações sobre todos esses aspectos foram levantadas, embora, por limitações de espaço, tenhase optado por apresentar apenas os dados mais relevantes e generalizáveis da pesquisa.

Os resultados expostos a seguir referem-se às seguintes questões: a) tipo de estabelecimento preferencialmente utilizado para adquirir FLV; b) grau de importância atribuído a diferentes critérios no momento da escolha do estabelecimento em que adquire
FLV; c) freqüência de aquisição de FLV; d) freqüência de consumo de diferentes FLV; e e) grau de importância a diferentes critérios no momento da escolha dos produtos FLV a serem adquiridos.

\section{RESULTADOS E DISCUSSÃO}

A primeira questão a ser apresentada referese aos principais locais onde os consumidores adquirem FLV na região pesquisada (Tabela 1). Os resultados indicaram que, nos principais centros urbanos da região central do Estado do Rio Grande do Sul (Santa Maria e Cachoeira do Sul), a maioria dos consumidores (59,77\%) tem optado por adquirir os produtos em supermercados ou hipermercados, seguidos das fruteiras e feiras livres. Somente em quarto lugar aparecem os pequenos mercados de bairro. Compras em feiras ecológicas e diretamente dos produtores rurais apresentam percentuais modestos em relação às demais alternativas. Por outro lado, nas cidades de menor porte (Quarta Colônia), a preferência é pela aquisição dos referidos produtos em fruteiras (54,29\%), seguidas dos hiper/supermercados (22,86\%).

Esses resultados eram de alguma forma esperados, tendo-se em vista que algumas pesquisas têm apontado uma supremacia dos super e hipermercados na preferência dos consumidores quando da aquisição de alimentos no mundo inteiro (KOHLS, 2004; JUNIOR, 1999; BELIK, 1995).

Com relação aos critérios de escolha do local para a compra dos produtos, a pesquisa identificou os aspectos mais importantes segundo a opinião dos entrevistados. Os resultados são apresentados na tabela 2 e referem-se a uma questão em que se perguntava qual o grau de importância de cada um dos critérios selecionados (constantes na tabela) no momento da escolha do local para a compra de FLV. As respostas foram indicadas em uma escala composta

Tabela 1 - Locais onde os entrevistados costumam adquirir frutas, legumes e verduras (FLV) na região central do Rio Grande do Sul, 2005 (número e percentagem de entrevistados).

\begin{tabular}{|c|c|c|c|c|c|c|c|c|}
\hline \multirow[b]{2}{*}{ Item } & \multicolumn{2}{|c|}{----Santa Maria---- } & \multicolumn{2}{|c|}{---Cachoeira do Sul--- } & \multicolumn{2}{|c|}{----Quarta Colônia---- } & \multicolumn{2}{|c|}{-----Total----- } \\
\hline & $\mathrm{N}^{\mathrm{o}}$ & $\%$ & $\mathrm{~N}^{\mathrm{o}}$ & $\%$ & $\mathrm{~N}^{\mathrm{o}}$ & $\%$ & $\mathrm{~N}^{\circ}$ & $\%$ \\
\hline Hiper/supermercados & 118 & 67,0 & 33 & 60,0 & 8 & 22,9 & 159 & 59,8 \\
\hline Fruteiras & 20 & 11,4 & 8 & 14,5 & 19 & 54,3 & 47 & 17,7 \\
\hline Feiras livres convencionais & 13 & 7,4 & 8 & 14,5 & 3 & 8,6 & 24 & 9,0 \\
\hline Minimercados & 16 & 9,1 & 4 & 7,3 & 2 & 5,7 & 22 & 8,3 \\
\hline Feiras ecológicas & 5 & 2,8 & 0 & 0,0 & 2 & 5,7 & 7 & 2,6 \\
\hline Direto do produtor & 4 & 2,3 & 2 & 3,6 & 1 & 2,9 & 7 & 2,6 \\
\hline
\end{tabular}

Fonte: Dados coletados pelos autores. 
Tabela 2 - Importância relativa de diferentes critérios utilizados na escolha do estabelecimento para a compra de frutas, legumes e verduras (FLV), em ordem decrescente de importância (número e percentagem de entrevistados que responderam "muito" ou "o mais" importante).

\begin{tabular}{|c|c|c|c|c|c|c|c|c|}
\hline \multirow[b]{2}{*}{ Critério } & \multicolumn{2}{|c|}{----Santa Maria---- } & \multicolumn{2}{|c|}{---Cachoeira do Sul--- } & \multicolumn{2}{|c|}{----Quarta Colônia---- } & \multicolumn{2}{|c|}{------Total------ } \\
\hline & $\mathrm{N}^{\mathrm{o}}$ & $\%$ & $\mathrm{~N}^{\mathrm{o}}$ & $\%$ & $\mathrm{~N}^{\mathrm{o}}$ & $\%$ & $\mathrm{~N}^{\mathrm{o}}$ & $\%$ \\
\hline Qualidade dos produtos & 172 & 97,7 & 54 & 98,2 & 35 & 100,0 & 261 & 98,1 \\
\hline Variedade de produtos & 151 & 85,8 & 42 & 76,4 & 32 & 91,4 & 225 & 84,6 \\
\hline Agilidade no check-out & 139 & 79,0 & 46 & 83,6 & 30 & 85,7 & 215 & 80,8 \\
\hline Preço dos produtos & 136 & 77,3 & 48 & 87,3 & 27 & 77,1 & 211 & 79,3 \\
\hline $\begin{array}{l}\text { Distribuição dos produtos no } \\
\text { local }\end{array}$ & 135 & 76,7 & 36 & 65,4 & 28 & 80,0 & 199 & 74,8 \\
\hline Segurança & 131 & 74,4 & 39 & 70,9 & 28 & 80,0 & 198 & 74,4 \\
\hline Confiança no estabelecimento & 132 & 75,0 & 34 & 61,8 & 31 & 88,6 & 197 & 74,1 \\
\hline Conforto do estabelecimento & 112 & 63,6 & 41 & 74,5 & 26 & 74,3 & 179 & 67,3 \\
\hline Proximidade da residência & 107 & 60,8 & 28 & 50,9 & 19 & 54,3 & 154 & 57,9 \\
\hline Facilidade de estacionamento & 94 & 53,4 & 25 & 45,4 & 14 & 40,0 & 133 & 50,0 \\
\hline Forma de pagamento & 67 & 38,1 & 25 & 45,4 & 16 & 45,7 & 108 & 40,6 \\
\hline Facilidade de entrega & 66 & 37,5 & 25 & 45,4 & 13 & 37,1 & 104 & 39,1 \\
\hline
\end{tabular}

Fonte: Dados coletados pelos autores.

pelas seguintes alternativas: o mais importante; muito importante; medianamente importante; pouco importante; nada importante. Isso significa que a cada critério correspondia uma pergunta e que todas elas eram independentes umas das outras. Os dados relacionados na tabela 2 referem-se ao somatório das respostas “o mais importante” e "muito importante”.

O primeiro aspecto a ressaltar é o fato de não ter havido grandes diferenças no padrão comportamental dos consumidores tanto nas maiores quanto nas menores cidades pesquisadas. Os cinco principais critérios de escolha do estabelecimento para compra de FLV, na opinião dos entrevistados, foram: 1) qualidade dos produtos; 2) variedade de produtos; 3) agilidade no check-out; 4) preço dos produtos; e 5) distribuição dos produtos no local.

Chama a atenção o fato de a política de preços do estabelecimento ter sido apontada somente como o quarto critério de decisão mais importante na visão dos consumidores. Por outro lado, aspectos de qualidade e variedade de produto obtiveram, respectivamente, $98,12 \%$ (primeiro lugar) e $84,59 \%$ (segundo lugar) de respostas como sendo os critérios mais importantes para a definição do local de compra de FLV.

Outro dado importante é o fato de a variável "agilidade no check-out” do estabelecimento ter sido considerada mais importante que o "preço dos produtos”. Esse dado evidencia a relevância da variável "tempo" para os consumidores no atual contexto, mesmo em cidades de pequeno e médio porte.

Uma outra questão formulada aos entrevistados e apresentada na tabela 3 teve por objetivo verificar a freqüência com que são efetuadas as compras de FLV. Esse é um aspecto importante, tendo-se em vista que são produtos que fazem parte da dieta diária de muitas famílias e que apresentam a característica de estragarem com relativa brevidade após serem comprados.

Os resultados indicam que $39,85 \%$ dos entrevistados optam por realizar suas compras de FLV uma vez por semana, 34,57\% duas vezes por semana, $14,29 \%$ três vezes por semana e 9,77\% diariamente. Essa informação, em conjunto com os dados anteriormente apresentados sobre importância da agilidade no checkout e variedade de produtos no estabelecimento, reforça a tese de que o fator tempo dedicado às compras passou a ser um aspecto bastante importante no processo de tomada de decisão dos consumidores. No caso, 58,63\% dos entrevistados disseram que vão duas ou mais vezes por semana a estabelecimentos comerciais para adquirir FLV. Esta informação indica ser esta uma atividade que consome tempo considerável dos consumidores urbanos. Estas foram as principais informações em termos de escolha dos estabelecimentos comerciais por parte dos entrevistados.

Do ponto de vista dos produtos em si, uma das questões levantadas foi a freqüência de consumo dos diversos produtos que compõem a cesta de FLV identificada na região. O resultado está na tabela 4 .

Nessa questão, perguntou-se com que freqüência o consumidor consome cada um dos produtos indicados, se: regularmente, eventualmente 
Tabela 3 - Freqüência de aquisição de frutas, legumes e verduras (FLV) (número e percentagem de entrevistados).

\begin{tabular}{|c|c|c|c|c|c|c|c|c|}
\hline \multirow{2}{*}{ Freqüência } & \multicolumn{2}{|c|}{----Santa Maria---- } & \multicolumn{2}{|c|}{---Cachoeira do Sul--- } & \multicolumn{2}{|c|}{----Quarta Colônia---- } & \multicolumn{2}{|c|}{------Total------ } \\
\hline & $\mathrm{N}^{\mathrm{o}}$ & $\%$ & $\mathrm{~N}^{\mathrm{o}}$ & $\%$ & $\mathrm{~N}^{\mathrm{o}}$ & $\%$ & № & $\%$ \\
\hline Não adquire & - & - & - & - & - & - & - & - \\
\hline Uma vez por mês & - & - & - & - & - & - & - & - \\
\hline Duas vezes por mês & 2 & 1,1 & - & - & 1 & 2,9 & 3 & 1,1 \\
\hline Três vezes por mês & - & - & - & - & 1 & 2,9 & 1 & 0,4 \\
\hline Uma vez por semana & 65 & 36,9 & 23 & 41,8 & 18 & 51,4 & 106 & 39,8 \\
\hline Duas vezes por semana & 63 & 35,8 & 17 & 30,9 & 12 & 34,3 & 92 & 34,6 \\
\hline Três vezes por semana & 32 & 18,2 & 4 & 7,3 & 2 & 5,7 & 38 & 14,3 \\
\hline Diariamente & 14 & 8,0 & 11 & 20,0 & 1 & 2,9 & 26 & 9,8 \\
\hline
\end{tabular}

Fonte: Dados coletados pelos autores.

ou nunca. Caso a resposta fosse "regularmente", desdobrava-se a pergunta em "diariamente" ou "não diariamente".

Um primeiro aspecto a ressaltar é que, dos vinte produtos que fazem parte da cesta de FLV pesquisada, apenas dois apresentam o consumo eventual superior ao consumo regular: o morango e a pêra. Os demais demonstraram uma expressiva regularidade no consumo.
Essa regularidade no consumo da maior parte das FLV é um dado importante quando se pretende desenvolver a produção desses produtos na região ou se pensar em estabelecer um comércio de FLV. Os dados indicam que, mesmo apresentando produção com forte componente sazonal, alguns produtos têm consumo regular, sinalizando que os consumidores já estão habituados a consumi-los ao longo de todo o ano.

Tabela 4 - Freqüência de consumo de frutas, legumes e verduras (FLV) na região central do Rio Grande do Sul, 2005 (número e percentagem de entrevistados).

\begin{tabular}{|c|c|c|c|c|c|c|c|c|c|c|c|c|}
\hline \multirow{2}{*}{ Produto } & \multicolumn{6}{|c|}{ Regularmente } & \multicolumn{2}{|c|}{ Eventualmente } & \multicolumn{2}{|c|}{----Nunca---- } & \multicolumn{2}{|c|}{-----Total----- } \\
\hline & $\mathrm{N}^{\mathrm{o}}$ & $\%$ & $\mathrm{~N}^{\mathrm{o}}$ & $\%$ & $\mathrm{~N}^{\mathrm{o}}$ & $\%$ & $\mathrm{~N}^{\mathrm{o}}$ & $\%$ & $\mathrm{~N}^{\mathrm{o}}$ & $\%$ & $\mathrm{~N}^{\circ}$ & $\%$ \\
\hline Cebola & 233 & 87,6 & 22 & 8,3 & 255 & 95,9 & 7 & 2,6 & 4 & 1,5 & 266 & 100,0 \\
\hline Alface & 190 & 71,4 & 58 & 21,8 & 248 & 93,2 & 10 & 3,8 & 8 & 3,0 & 266 & 100,0 \\
\hline Tempero Verde & 200 & 75,2 & 46 & 17,3 & 246 & 92,5 & 10 & 3,8 & 10 & 3,8 & 266 & 100,0 \\
\hline Tomate & 210 & 79,0 & 36 & 13,5 & 246 & 92,5 & 18 & 6,8 & 2 & 0,8 & 266 & 100,0 \\
\hline Laranja & 122 & 45,9 & 121 & 45,5 & 243 & 91,4 & 19 & 7,1 & 4 & 1,5 & 266 & 100,0 \\
\hline Alho & 195 & 73,3 & 32 & 12,0 & 227 & 85,3 & 18 & 7,1 & 21 & 7,6 & 266 & 100,0 \\
\hline Bergamota & 104 & 39,1 & 105 & 39,5 & 209 & 78,6 & 49 & 18,4 & 8 & 3,0 & 266 & 100,0 \\
\hline Mandioca & 63 & 23,7 & 141 & 53,0 & 204 & 76,7 & 52 & 19,6 & 10 & 3,8 & 266 & 100,0 \\
\hline Cenoura & 82 & 30,8 & 106 & 39,8 & 188 & 70,7 & 70 & 26,3 & 8 & 3,0 & 266 & 100,0 \\
\hline Rúcula & 93 & 35,0 & 92 & 34,6 & 185 & 69,6 & 41 & 15,4 & 40 & 15,0 & 266 & 100,0 \\
\hline Beterraba & 56 & 21,0 & 108 & 40,6 & 164 & 61,7 & 81 & 30,4 & 21 & 7,9 & 266 & 100,0 \\
\hline Couve & 52 & 29,6 & 110 & 41,4 & 162 & 60,9 & 70 & 26,3 & 34 & 12,8 & 266 & 100,0 \\
\hline Limão & 85 & 32,0 & 74 & 27,8 & 159 & 59,8 & 65 & 24,4 & 42 & 15,8 & 266 & 100,0 \\
\hline Couve-Flor & 46 & 17,3 & 105 & 39,5 & 151 & 56,8 & 79 & 29,7 & 36 & 13,5 & 266 & 100,0 \\
\hline Radicci & 59 & 22,2 & 82 & 30,8 & 141 & 53,0 & 52 & 19,6 & 73 & 27,4 & 266 & 100,0 \\
\hline Pepino & 28 & 10,5 & 100 & 37,6 & 128 & 48,1 & 76 & 28,6 & 62 & 23,3 & 266 & 100,0 \\
\hline Pêssego & 26 & 9,8 & 93 & 35,0 & 119 & 44,7 & 109 & 41,0 & 38 & 14,3 & 266 & 100,0 \\
\hline Uva & 32 & 12,0 & 85 & 32,0 & 117 & 44,0 & 121 & 45,5 & 28 & 10,5 & 266 & 100,0 \\
\hline Morango & 9 & 3,4 & 63 & 23,9 & 72 & 27,1 & 155 & 58,3 & 39 & 14,7 & 266 & 100,0 \\
\hline Pêra & 6 & 2,3 & 53 & 19,9 & 59 & 22,2 & 95 & 35,7 & 112 & 42,1 & 266 & 100,0 \\
\hline
\end{tabular}

Fonte: Dados coletados pelos autores. 
Os dados também permitem classificar os produtos em três grupos. Um primeiro grupo é formado por produtos cujo consumo se dá com boa regularidade (indicados como consumidos "regulamente” por mais de $80 \%$ dos entrevistados) e que inclui cebola (95,86\%), alface $(93,23 \%)$, tempero verde $(92,48 \%)$, tomate (92,48\%), laranja (91,35\%) e alho (85,34\%). Um segundo grupo é formado por produtos cuja indicação de que são consumidos regularmente atingiu uma faixa entre 50 e $80 \%$ das respostas: bergamota (78,57\%), mandioca (76,69\%), cenoura (70,68\%), rúcula $(69,55 \%)$, beterraba (61,65\%), couve (60,90\%), limão (59,77\%), couve-flor (56,77\%) e radicci $(53,00 \%)$. E um terceiro grupo é composto por aqueles produtos cujas indicações de regularidade no consumo se deram abaixo de $50 \%$ : pepino $(48,12 \%)$, pêssego $(44,74 \%)$, uva $(43,98 \%)$, morango (27,08\%) e pêra (22,18\%).

Por fim, a pesquisa pretendia identificar quais os aspectos relacionados aos produtos em si são mais importantes na tomada de decisão de compra de FLV pelos consumidores. O método de pergunta relacionado a essa questão foi idêntico ao apresentado na tabela 2, assim como a forma de tabulação dos dados para hierarquizar os fatores mais importantes. As respostas encontram-se na tabela 5 .

$\mathrm{O}$ aspecto considerado mais importante em todas as cidades foi a "aparência” dos produtos, seguido pelo "sabor" que os consumidores imaginam que os mesmos possuem. Em terceiro lugar, foi citado o "preço" dos produtos, seguido pelos "aspectos nutricionais” e, em quinto lugar, pela "durabilidade” prevista após a compra. As respostas dessa questão reforçam os dados anteriores sobre a importância da qualidade para a definição de compra dos produtos e estão em conformidade com as tendências do mercado de alimentos expostas por JÚNIOR (1999). Quatro dos cinco principais critérios podem ser considerados atributos intrínsecos de qualidade: aparência, sabor, aspectos nutricionais e durabilidade.

\section{CONCLUSÕES}

Os resultados da pesquisa indicam que, nos principais centros urbanos da região central do Estado do Rio Grande do Sul, a maioria dos consumidores tem optado por adquirir FLV em supermercados ou hipermercados. Fatores ligados à qualidade e variedade de produtos, bem como à agilidade no momento da compra, sobrepõem-se aos preços dos produtos como critérios de escolha dos consumidores em relação ao estabelecimento em que adquirirão os produtos. Estas informações indicam a importância e relevância que os consumidores atribuem a duas variáveis: qualidade e tempo, no processo de compra de FLV.

Isso se confirma também pelos dados que demonstram que, dos cinco principais aspectos dos produtos considerados pelos consumidores na escolha de FLV, quatro referem-se à qualidade (aparência, sabor, aspectos nutricionais e durabilidade), sendo que o preço aparece apenas em terceiro lugar, depois de aparência e de sabor.

Este aspecto pode estar relacionado a duas questões. Primeiro, ao fato de que FLV são produtos altamente diversificados e perecíveis, o que faz com que a qualidade dos mesmos possa variar significativamente entre os estabelecimentos e, segundo, ao fato de que, por serem perecíveis, os consumidores necessitam adquiri-los com maior freqüência, como foi identificado na pesquisa, o que implica maior dispêndio de tempo com esses produtos.

Tabela 5 - Importância relativa de diferentes critérios utilizados para a escolha dos produtos frutas, legumes e verduras (FLV), em ordem decrescente de importância (número e percentagem de entrevistados que responderam “muito” ou “o mais” importante).

\begin{tabular}{|c|c|c|c|c|c|c|c|c|}
\hline \multirow{2}{*}{ Critério } & \multicolumn{2}{|c|}{----Santa Maria---- } & \multicolumn{2}{|c|}{----Cachoeira do Sul---- } & \multicolumn{2}{|c|}{----Quarta Colônia---- } & \multicolumn{2}{|c|}{------Total------- } \\
\hline & $\mathrm{N}^{\circ}$ & $\%$ & $\mathrm{~N}^{\mathrm{o}}$ & $\%$ & $\mathrm{~N}^{\circ}$ & $\%$ & $\mathrm{~N}^{\mathrm{o}}$ & $\%$ \\
\hline Aparência & 161 & 91,5 & 55 & 100,0 & 35 & 100,0 & 251 & 94,4 \\
\hline Sabor & 146 & 83,0 & 51 & 92,7 & 32 & 91,4 & 229 & 86,1 \\
\hline Preço & 127 & 72,2 & 51 & 92,7 & 27 & 77,1 & 205 & 77,1 \\
\hline Aspectos nutricionais & 134 & 76,1 & 43 & 78,2 & 27 & 77,1 & 204 & 76,7 \\
\hline Durabilidade & 128 & 72,7 & 43 & 78,2 & 28 & 80,0 & 199 & 74,8 \\
\hline Acondicionamento & 134 & 76,1 & 37 & 67,3 & 21 & 60,0 & 192 & 72,2 \\
\hline Cheiro & 126 & 71,6 & 34 & 61,8 & 21 & 60,0 & 181 & 68,0 \\
\hline Textura & 122 & 69,3 & 35 & 63,6 & 22 & 62,9 & 179 & 67,3 \\
\hline Caract. do processo* & 114 & 64,8 & 38 & 69,1 & 21 & 60,0 & 173 & 65,0 \\
\hline Origem definida & 109 & 61,9 & 32 & 58,2 & 19 & 54,3 & 160 & 60,2 \\
\hline
\end{tabular}

Fonte: Dados coletados pelos autores. 
Assim, qualidade e tempo se tornam variáveis críticas e quase em igual nível de importância para os consumidores.

Por fim, a pesquisa identificou os produtos com maior potencial de consumo e, consequentemente, de produção na região. O conjunto de produtos consumidos de forma mais regular e constante durante todo o ano são: cebola, laranja, tempero verde, tomate, alho e alface.

\section{AGRADECIMENTOS}

Os autores agradecem à Fundação de Amparo à Pesquisa do Rio Grande do Sul (FAPERGS) pelo financiamento desta pesquisa através do Edital PROCOREDES, de 2004.

\section{REFERÊNCIAS}

ADLER, M.K. A moderna pesquisa de mercado. 2.ed. São Paulo: Pioneira, 1971. 138p.

BELIK, W. Reestruturação industrial e estratégias dos grupos agro-alimentares no Brasil. In: RAMOS, P.; REYDON, B.P. (Orgs). Agropecuária e agroindústria no Brasil: ajuste, situação atual e perspectivas. Campinas: ABRA, 1995. p.107-126.

FOWLER, F.J. Survey research metods. 2.ed. Newbury Park: Sage Publications, 1993. 156p.
GAINS, N. The repertory grid approach. In: MACFIE, H.J. H.; THOMSON, D.M.H. (Coords.). Measurement of food preference. London: Blackic Academic \& Professional, 1994 320p.

IBGE (Rio de Janeiro - RJ). Censo Demográfico 2000. Disponível: site IBGE (2000). Acesso: ago. 2005. On line. Disponível em: http://www.sidra.ibge.gov.br/bda/tabela/ listabl.asp?z=t\&o=1\&i $=\mathrm{P} \& \mathrm{e}=\mathrm{l} \& \mathrm{c}=200$.

JUNIOR, O.M. A globalização e a indústria alimentar: um estudo a partir das grandes empresas. São Paulo: FAPESP, 1999. 194p.

KERLINGER, F.N. Metodologia da pesquisa em ciências sociais: um tratamento conceitual. São Paulo: EPU/ EDUSP, 1980. 377p.

KOHLS, V.K. As ênfases estratégicas de empresas agroalimentares: estudo de casos na região de PelotasRS. Tese (Doutorado em Administração) - Escola de Administração, PPGA/UFRGS, 2004. 238p.

LIMA FILHO, D.O. et al. Comportamento alimentar do consumidor idoso. In: ENCONTRO DA ASSOCIAÇÃO NACIONAL DE PÓS-GRADUAÇÃO E PESQUISA EM ADMINISTRAÇÃO, 28., 2004, Curitiba, PR. Anais... Rio de Janeiro: ANPAD, 2004. p.1-11.

PAULILLO, L.F.; PESSANHA, L. Segurança alimentar e políticas públicas: conexões, implicações e regionalização. In: PAULILLO, L.F.; ALVES, F. Reestruturação agroindustrial - políticas públicas e segurança alimentar regional. São Carlos: Edufscar, 2002. p.17-56 